\title{
Régulation de l'expression du gène c-myc
}

\section{Un blocage dans l'élongation des transcrits}

Les nouvelles
de ce numéro ont été préparées par : A. Kahn J.-C. Kaplan J.-C. Dreyfus
1. Remmers EF, Yang JQ Marcu KB. A negative transcriptional control element located upstream of the murine $c$-myc gene. $E M B O$ $J 1986$; 5 : 899-904.

2. Chung J, Sinn E, Reed RR, Leder P. Trans-acting elements modulate expression of the human $c$-myc gene in Burkitt lymphoma cells. Proc Natl Acad Sci USA 1986; 83 : 7918-22.

3. Bentley DL, Groudine M. A block to elongation is largely responsible for decreased transcription of $c$-myc in differentiated HL.60 cells. Nature 1986 ; 321 : 702-6.

4. Eick D, Bornkamm GW. Transcriptional arrest within the first exon is a fast control mechanism in $c$-myc gene expression. Nucleic Acids Res 1986 ; 14 : 8331-46.

5. Nepveu A, Marcu KB. Intragenic pausing and anti-sense transcription within the murine $c$-myc locus. EMBO J $1986 ; 5$ : 2859-65.

6. Bentley DL, Groudine M. Novel promotor upstream of the human $c$-myc gene and regulation of $c$-myc expression in $\beta$ cell lympho-
L'oncogène $c$-myc est exprimé de façon variable selon l'état prolifératif des cellules, faiblement dans des cellules quiescentes et fortement dans des cellules en croissance rapide. Dans les cellules HL.60 (dérivées d'une leucémie aiguë promyélocytaire humaine), le gène $c$-myc est amplifié et très activement transcrit. Lorsque la différenciation de ces cellules en granulocytes est induite par un traitement à l'acide rétinoïque ou au DMSO (diméthylsulfoxyde), la concentration du messager $c$-myc diminue considérablement en quelques dizaines de minutes. Les mécanismes de contrôle de l'expression du gène $c$-myc sont probablement nombreux, transcriptionnels et post-transcriptionnels. Nous avons ainsi commenté dans ces colonnes l'importance de la modulation de la stabilité de l'ARNm $c$-myc dans la régulation de l'expression de cet oncogène (voir $\mathrm{m} / \mathrm{s} n^{\circ} 5$, vol. $\left.2, p .286\right)$. Dans les lymphomes de Burkitt, un des allèles de $c$-myc est réarrangé et se retrouve à proximité des gènes codant pour les immunoglobulines, ce qui en active fortement l'expression, par un mécanisme transcriptionnel ou/et par une augmentation de la stabilité du messager du gène $c$-myc réarrangé. L'hyperexpression de l'allèle réarrangé conduit à l'inhibition de l'expression de l'allèle normal, ce qui fait supposer que le produit $c$-myc pourrait être un régulateur négatif de l'expression de son propre gène. Cette rétro-inhibition porte sur l'initiation de la transcription au niveau des deux promoteurs $\left(P_{1}\right.$ et $\left.P_{2}\right)$ contrôlant la synthèse des espèces majoritaires de messagers. On ne connaît pas encore le mécanisme en cause, mais un "silencer " a été décrit en amont du gène de souris [1], trois autres séquences de régulation négative étant mises en évidence au niveau du gène humain, en amont des promoteurs $P_{1}$ et $P_{2}$ et dans le premier intron [2]. Une séquence de régulation positive existerait également en amont des promoteurs $P_{1}$ et $P_{2}$ [2]. Le mécanisme de la diminution de synthèse de nouveaux transcrits $c$-myc lors de l'induction de la différenciation terminale des cellules HL.60 n'est cependant pas une diminution de l'initiation de la transcription du gène par de nouvelles molécules d'ARN polymérase II, mais un blocage de l'élongation des transcrits par l'ARN polymérase à la jonction du premier exon et du premier intron $[3,4,5]$. En d'autres termes, le nombre de molécules d'ARN polymérase engagées dans la transcription du gène ne diminue pas au niveau du premier exon, mais chute brutalement au-delà. Des protéines labiles, dont la concentration est supposée augmenter lors du traitement des cellules HL.60 par des inducteurs de différenciation, seraient impliquées dans ce blocage de l'élongation des transcrits. Dans certaines cellules dérivées de lymphome de Burkitt, le blocage transcriptionnel au niveau de l'extrémité du premier exon est supprimé ; cela pourrait être la conséquence des fréquentes mutations ponctuelles qui s'accumulent au niveau du premier exon de gènes $c$-myc réarrangés [6].

En conclusion, l'expression du gène $c$-myc est régulée de manière extrêmement complexe à différents niveaux. L'initiation de la transcription est contrôlée positivement et négativement. L'élongation des transcrits est soumise à un blocage variable au niveau de la jonction entre le premier exon et le premier intron, un tel mécanisme de contrôle transcriptionnel agissant sur l'élongation étant décrit ici pour la première fois chez les eucaryotes. Enfin, la stabilité des transcrits est soumise à une modulation rapide.
A. $\mathbf{K}$. 


\section{L'athérome : une maladie tumorale?}

Trois séquences d'événements distincts sont associées au développement de la plaque d'athérome : l'accumulation des lipides, la thrombose, la prolifération cellulaire. Jusqu'ici ce sont surtout les deux premiers aspects qui avaient retenu l'attention. L'article que viennent de publier Penn et al. [1] donne la vedette à la composante cellulaire en montrant que l'ADN des cellules musculaires lisses des plaques d'athérome possède les mêmes vertus transformantes que celui d'une cellule cancéreuse. Autrement dit, la plaque d'athérome pourrait bien être une minitumeur de la paroi vasculaire.

L'origine de ce travail repose sur la découverte en 1973 de l'origine monoclonale de la prolifération de ces cellules, qui sont l'élément cellulaire prédominant dans la plaque d'athérome [2]. Celle-ci apparaissait comme résultant d'un événement mutationnel somatique, viro- ou chimio-induit, survenu dans l'ADN d'une seule cellule. Cette hypothèse devait être par la suite confortée par toute une série de travaux démontrant que l'administration, à des coquelets, d'agents cancérigènes chimiques (diméthylbenzanthracène, benzopyrène) ou viraux (virus de Marek) induisait l'apparition de plaques fibro-musculaires sur l'aorte abdominale et thoracique. Il ne restait plus qu'à démontrer que l'ADN des plaques d'athérome avait des propriétés anormales. C'est ce qu'ont fait Penn et al. [1] en recourant à la méthodologie désormais classique de la recherche en oncologie moléculaire [3]. Les résultats expérimentaux sont impressionnants : (a) l'ADN des plaques contient des séquences capables de transformer les cellules NIH 3T3 avec une efficacité deux à huit fois supérieure au bruit de fond ; (b) ce pouvoir transformant peut être reproduit lors de cycles de transfection successifs ; (c) l'ADN des cellules transformées corres$\mathrm{m} / \mathrm{s} n^{\circ} 3 \mathrm{ool} .3$, mars 87 pond bien à l'ADN humain car il hybride avec une sonde de la famille répétitive dispersée Alu ; (d) l'ADN transformant n'hybride avec aucune séquence de la famille des oncogènes ras; (e) l'ADN des cellules transformées est tumorigène chez la souris nude; (f) l'ADN des tumeurs induites chez la souris nude hybride avec une sonde Alu humaine.

Reste à démontrer que l'activation de l'oncogène mis en évidence par Penn et al. [1] dans des plaques d'athérome coronarien est un phénomène primaire, existant dans le tissu de départ, et non un phénomène artéfactuel dû à un réarrangement génique survenant au cours des différentes étapes de la transfection, comme cela s'avère en fait fréquent [4]. Il faut bien dire que les preuves définitives de l'existence de l'oncogène activé dans les cellules musculaires lisses des plaques athéromateuses manquent encore. S'il en était cependant bien ainsi, cela signifierait que le développement de l'athérome comporterait une composante cellulaire - une mutation somatique - dont il resterait alors à apprécier les rapports de causalité ou de coïncidence avec les facteurs prédisposants que sont les habitudes alimentaires et les concentrations relatives des différentes lipoprotéines. L'athérosclérose étant une maladie multifactorielle, on peut concevoir un modèle dans lequel son développement serait le résultat de l'interaction entre deux types de facteurs favorisants : une prédisposition génétique à des mutations somatiques des cellules de la paroi vasculaire d'une part, les anomalies plasmatiques génétiques ou secondaires aux habitudes alimentaires d'autre part. Alternativement, les désordres plasmatiques pourraient être eux-mêmes à l'origine des mutations somatiques activant un " oncogène " de la paroi artérielle.

$$
\text { J.-C. K., A. K. }
$$

1. Penn A, Garte SJ, Waren L, Nesta D, Mindich B. Transforming gene in human atherosclerotic plaque DNA. Proc Natl Acad Sci USA $1986 ; 83$ : 7951-5.

2. Benditt EP, Benditt JM. Evidence for a monoclonal origin of human atherosclerotic plaques. Proc Natl Acad Sci USA 1973; 70 : 1753-6.

3. Stéhelin D. Les oncogènes cellulaires, clés de la cancérogenèse. médecine/sciences $1985 ; 1$ : 12-6.

4. Young D, Waitches G, Birchmeier C Fasano C, Wigler $M$. Isolation and characterization of a new cellular oncogene encoding a protein with multiple potential transmembrane domains. Cell $1986 ; 45$ : 711-9.

\section{D曰 BRÈVES}

- Polyadénylation, coupure endonucléolytique de l'extrémité $3^{\prime}$ des transcrits et fin de transcription seraient des phénomènes liés. Un gène d' $\alpha$ globine dont le signal de polyadénylation AATAAA est muté en AATAAC donne des transcrits non polyadénylés et allongés au-delà du site normal de fin de transcription. Ces anomalies entraînent un syndrome $\alpha$-thalassémique.

[Whitelaw E, Proudfoot N. $E M B O \mathrm{~J} 1986$; 5 : 2915-22.]

Un nouvel exemple de vaccination contre un rétrovirus, le virus de Friend, vient d'être rapporté. Ce rétrovirus provoque une érythroleucémie murine. Des souris vaccinées par un virus de la vaccine recombiné par l'introduction du gène env du virus de Friend, codant pour la glycoprotéine de surface, développent une immunité spécifique et sont protégées contre la survenue d'une leucémie après infection par le virus leucémogène. Cette recherche sur les vaccins antirétroviraux trouve toute son actualité dans le cadre des efforts actuels pour développer une vaccination efficace contre le SIDA. [Earl PL. Science 1986 ; 234 728-31.]
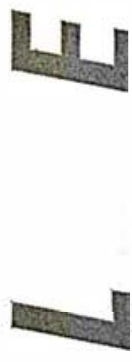

\section{.}

.

